\title{
ANTI-PLANE SURFACE DISTURBANCE
}

\author{
M. Azram \\ Department of Science in Engineering, Kulliyyah of Engineering \\ International Islamic University Malaysia, Jalan Gombak, 53100 Kuala Lumpur
}

\begin{abstract}
Solution to a problem of half-space subjected to anti-plane surface disturbance via Cagniard-de Hoop method has been investigated. The solution is very much dependent on the choice of branch cuts, branches, and their behavior along the appropriate selected contour. Investigations show that the contour, which simplifies the inversion integral, may or may not exist in some quadrants of $\xi$-plane and is very much dependent on the position of the point $\xi$. Consequently, the solution is the Hankel function of first kind $\frac{i}{2} H_{0}^{(1)}(k r)$.
\end{abstract}

Keywords: Cagniard-de Hoop method. Inversion Integral. Hankel function. Anti-plane Shear. Waves propagation. Fourier Transform.

\section{INTRODUCTION}

Surface disturbances give rise to waves. Waves are a ubiquitous and important feature of the physical world, and throughout the history it has been a major challenge to understand them. They can propagate on surfaces of solids and of fluids. Chemical waves control the heartbeat. In recent years, elastic waves transmitted through the human body have been used for medical diagnoses and therapy. Waves propagation as one of basic phenomena in solids is of both theoretical and practical importance. Waves propagation in elastic solids is a very broad and attractive research field. The study of waves propagation in elastic solids has a long and distinguished history ${ }^{[1]}$.

Cauchy and Poisson have studied the propagation of a disturbance in an elastic aether as early as middle of the $19^{\text {th }}$ century. Their contribution and work is now, generally known as the "theory of elasticity". Early investigations on the propagation of waves in elastic solids have been carried out by Cauchy, Clebsch, Christoffel, Green, Lame', Ostrogradsky, Poisson, and Stokes. This work has been briefly discussed in the book "The mathematical theory of elasticity" written by Love $^{[2]}$. Another classical book is by Timoshenko ${ }^{[3]}$. Lamb, Love, and Rayleigh contributions in this area are of significant importance.

Many professionals in engineering, physics, geology, mathematics, and life science encounter problems requiring an understanding of elastic waves. The study of wave propagation in elastic solids have stimulating and wide-ranging engineering applications, such as earthquake phenomena, nuclear explosion, seismology ${ }^{[4-6]}$, structure under high rate of loading, high-speed machinery, ultrasonics and piezoelectric, material science, applied mechanics, in-situ safety and reliability control of complex structural components by acoustic emission, quantitative non-destructive material testing by ultrasonics, dynamic fracture mechanics, fluid mechanics, aerodynamics, anistrophy, geophysics, and elastodynamics $^{[7]}$, etc. For other significant contributions in the field of engineering, reader is encouraged to consult references ${ }^{[1,8-13]}$.

Parallel to the study of waves in elastic solids, the propagation of waves has also been investigated within the context of applied mathematics, electromagnetic theory and acoustics ${ }^{[11,14-19]}$.

Exposure to mathematical formulation of governing equations of wave motion is essential to understand the basic principles of wave motion in solids. A wide range of problems in this area has been studied based on mathematical, physical, experimental and numerical approaches. Consequently, large numbers of methods have been developed in this domain. Currently, the most commonly used methods are finite differences, finite elements ${ }^{[20,21]}$, spectral methods and boundary elements, soap-film method, photo elastic method, integral transform, discrete Fourier transform, Maliuzhinets' method, numerical modeling, Wienerhopf technique, and Cagniard-de Hoop method. Numerical modeling of elastic waves is important in many applications, such as geophysics, non-destructive testing, and fluid-structure interaction. The Wienerhopf technique ${ }^{[22]}$ has, since its invention in 1931, proved to be an elegant method in engineering and mathematical physics. Advantage of this method is that it provides an exact solution of integral equations. Geophysics, elastic and electromagnetic waves, diffraction of acoustic, fracture mechanics etc. are in the domain of its applications. Parallel to Wiener-hopf technique, an immensely important method is the Cagniard-de Hoop method. It is within the context of the application of Laplace transform but a considerable amount of complex analysis is required. This method was firstly established by ${ }^{[6]}$ and then modified by de Hoop $^{[23]}$.

\section{BACKGROUND}

Achenbach ${ }^{[1]}$, has used the Cagniard-de Hoop Method to solve a problem of half-space subjected to anti-plane surface disturbance i.e., he considered the half-space y $\geq 0$ subjected to the surface disturbance of the form (at $\mathrm{y}=0$ )

$t_{y z}=m T(x) e^{i w t}$ 
Where $t_{y z}$ is the Shear Stress, $\mu$ is elastic constant,

$T(x)$ is the strain, $\omega$ is the circular velocity, and $t$ is the time. The anti-plane shear disturbance generates a deformation which is governed by

$$
\frac{\mathbb{I}^{2} w}{\mathbb{I} x^{2}}+\frac{\mathbb{I}^{2} w}{\operatorname{I} y^{2}}=\frac{1}{c_{T}^{2}} \frac{\mathbb{I}^{2} w}{\mathbb{I} t^{2}}
$$

Where $w(x, y, t)$ is the displacement in $z$-direction and $c_{T}$ is the transverse velocity. If the surface disturbance has been operating for a long time, it is reasonable to assume that a steady state has been reached, and we may consider the displacement in the form

$w(x, y, t)=w(x, y) e^{i w t}$

The governing equation for $w(x, y)$ follows from (2) as a boundary value problem

$$
\begin{aligned}
& \frac{\mathbb{I}^{2} w}{\mathbb{I} x^{2}}+\frac{\mathbb{I}^{2} w}{\mathbb{I} y^{2}}+k_{T}^{2} w=0 \\
& \left.\frac{\mathbb{I} w}{\mathbb{I} y}\right|_{y=0}=T(x)
\end{aligned}
$$

where $k_{T}=\frac{w}{c_{T}}$ is the wave number, exponential

Fourier transform along with inverse transform are defined as;

$$
\begin{aligned}
& f^{*}(x)=\grave{\mathrm{O}}_{-}^{¥} e^{i x x} f(x) d x \\
& f(x)=\frac{1}{2 p} \grave{\mathrm{o}}_{-¥}^{¥} e^{-i x x} f^{*}(x) d x
\end{aligned}
$$

Exponential Fourier transform (5) applied to (4) yields;

$$
w^{*}(x, y)=-\frac{T^{*}(x)}{\sqrt{x^{2}-k_{T}^{2}}} e^{-\sqrt{x^{2}-k_{T}^{2}} y}
$$

where $T^{*}(x)$ is the Fourier transform of $T(x)$. Since the solution $w(x, y)$ is required to be bounded as $\mathrm{y} \rightarrow \infty$, hence, $w^{*}(\xi, y)$ in (7) is made bounded as $\mathrm{y} \rightarrow \infty$ by making the multiple-valued function $\sqrt{x^{2}-k_{T}^{2}}$ as single-valued by introducing the branch cuts emanating from the branch points $\xi= \pm k_{T}$. Branches are chosen such that $\operatorname{Re}\left(\sqrt{x^{2}-k_{T}^{2}}\right)$ is non-negative on the real $\xi$ axis. In addition, we choose $\sqrt{x^{2}-k_{T}^{2}}$ positive for $\operatorname{Re}(\xi)>k_{T}, \operatorname{Im}(\xi)=0$. The exact evaluation is carried out by a specific choice of $T(x)=\delta(x)$, where $\delta(x)$ is the Dirac delta function, which yields $T^{*}(\xi)=1$.

Consequently, the inverse Fourier transform of (7) yields the inversion integral

$$
w(x, y)=-\frac{1}{2 p} \grave{c}_{c} \frac{1}{\sqrt{x^{2}-k_{T}^{2}}} e^{-i x x-\sqrt{x^{2}-k_{T}^{2}} y} d x
$$

Where $\mathrm{c}$ is parallel but above and below to the negative and positive real axis respectively, as shown in Fig. 1.

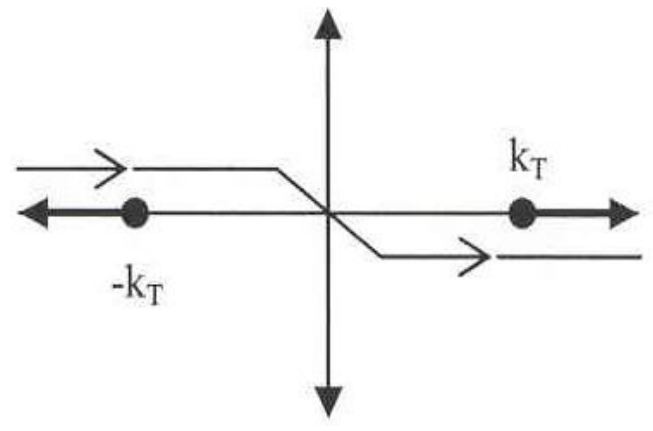

Fig. 1: Path in the $x$-plane.

With the introduction of $x=r \cos \theta$ and $y=r \sin \theta$, the integral equation (8) reduces as

$$
w(x, y)=\frac{-1}{2 p} \mathrm{Ò}_{c} \frac{1}{\sqrt{x^{2}-k_{T}^{2}}} e^{-i r\left\{x \cos q-i \sqrt{x^{2}-k_{T}^{2}} \sin q\right\}} d x
$$

This inversion integral is equivalent to the most often used inversion integral in elastodynamics given by

$$
w(x, y)=\underset{c}{\mathrm{O}} f(r, q, x) e^{i r\left\{x \cos q-i \sqrt{x^{2}-k_{T}^{2}} \sin q\right\}} d x
$$

where the contour $c$ is in the complex $\xi$-plane.

Application of the Cagniard-de Hoop method was the standard approach to find the solution, in which focus was to search a contour $c_{1}$ such that whenever $\xi$ is on $c_{1}$,

$S=x \cos q-i \sqrt{x^{2}-k_{T}^{2}} \sin q$.

This equation can be solved for $x$ as:

$x=S \cos q \pm i \sqrt{S^{2}-k_{T}^{2}} \sin q$, which yields the

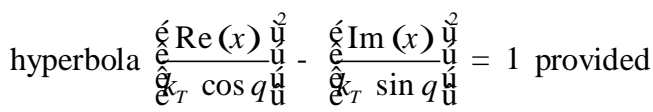

$S^{2}-k_{T}^{2} 30 \quad$ with $S$ to be real.

The required contour was branch of this hyperbola that lies in the right- half of the $\xi$ - plane as shown in Fig. 2. Consequently, the multiple valued function $\sqrt{x^{2}-k_{T}^{2}}$ was made single-valued function by introducing branch cuts emanating from the branch points $\pm k_{T}$ and going to the left and right along the real axis. The branch was chosen which has positive real part for $\xi$ on $c_{1}$. The ultimate solution was the Hankel function of second kind $w(x, y)=\frac{i}{2} H_{0}^{(2)}\left(k_{T} r\right)$.

Noble ${ }^{[22]}$ considered the same inversion integral with the assumption that $k_{T}$ is complex and $\xi=-k_{T} \cos (\theta+i t)$, where $-\infty<t<\infty$. 


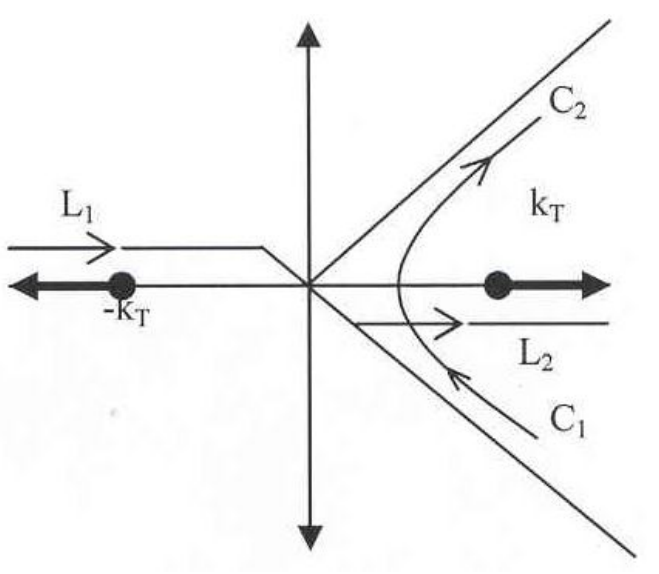

Fig. 2: Change of contour in the $\xi$-plane.

Consequently, the solution was

$\frac{i}{2} H_{0}^{(1)}\left(k_{T} r\right)$ if $k_{T}$ has small positive imaginary part and

$\frac{i}{2} H_{0}^{(2)}\left(k_{T} r\right)$ if $k_{T}$ has small negative imaginary part.

Both Achenbach ${ }^{[1]}$ as well as Noble ${ }^{[22]}$ searched the solution(s) by simplifying the inversion integral. Solution was very much dependent on the choice of branch cuts, branches, and their behaviors along the appropriate selected contour. Different modes of solution obtained for the same problem required further investigations of the inversion integral and the corresponding solution.

\section{INVESTIGATION AND DISCUSSION}

Consider the multiple valued function $f(x)=\sqrt{x^{2}-k_{T}^{2}}$ with two of its branches $f_{1}(x)$ and $f_{2}(x)$ defined by

$f_{1}(x)=\sqrt{r_{1} r_{2}} e^{\frac{i\left(q_{1}+q_{2}\right)}{2}}$

Where

$r_{1}=\left|x-k_{T}\right|>0, r_{2}=\left|x+k_{T}\right|>0$,

$q_{1}=\arg \left(x-k_{T}\right) ; 0<q_{1}<2 p$,

$q_{2}=\arg \left(x+k_{T}\right) ;-p<q_{2}<p$.

$f_{2}(x)=\sqrt{r_{1} r_{2}} e^{\frac{i\left(q_{1}+q_{2}\right)}{2}}$

Where

$r_{1}=\left|x-k_{T}\right|>0, r_{2}=\left|x+k_{T}\right|>0$,

$q_{1}=\arg \left(x-k_{T}\right) ; 2 p<q_{1}<4 p$,

$q_{2}=\arg \left(x+k_{T}\right) ;-p<q_{2}<p$.

Now, let us investigate the mapping properties of $f_{1}(\xi)$ : If $\xi$ lies in the first quadrant then; $\max \left(q_{1}+q_{2}\right)=p$

and $\min \left(q_{1}+q_{2}\right)=0$, which yields that $0<\left(q_{1}+q_{2}\right) / 2<p / 2$, concluding that $1^{\text {st }}$ quadrant maps into $1^{\text {st }}$ quadrant. If $\xi$ lies in the second quarter then; $\quad \max \left(q_{1}+q_{2}\right)=2 p$ and $\min \left(q_{1}+q_{2}\right)=p$ which yields $p / 2<\left(q_{1}+q_{2}\right) / 2<p$, concluding that $2^{\text {nd }}$ quadrant maps into $2^{\text {nd }}$ quadrant. If $\xi$ lies in the third quarter then; $\max \left(q_{1}+q_{2}\right)=p \quad$ and $\min \left(q_{1}+q_{2}\right)=0, \quad$ which yields that $0<\left(q_{1}+q_{2}\right) / 2<p / 2$, concluding that $3^{\text {rd }}$ quadrant maps into $1^{\text {st }}$ quadrant. Similarly; If $\xi$ lies in the fourth quarter then; $\quad \max \left(q_{1}+q_{2}\right)=2 p$ and $\min \left(q_{1}+q_{2}\right)=p, \quad$ which yields $p / 2<\left(q_{1}+q_{2}\right) / 2<p$, concluding that $4^{\text {th }}$ quadrant maps into $2^{\text {nd }}$ quadrant.

Line segment $-k<\operatorname{Re}(\xi) \leq 0$ and $0 \leq \operatorname{Re}(\xi)<k$ maps into $0<\operatorname{Im}(\xi) \leq k$ (twice) i.e., $-k<\operatorname{Re}(\xi)<k$ maps into $0<\operatorname{Im}(\xi) \leq k$. The positive and negative imaginary axes map into the ray $\operatorname{Im}(\xi)>k$.

It is interesting to observe that $f_{1}(x)=-f_{2}(x)$; hence, we may also conclude that $f_{2}(x)$ maps; $1^{\text {st }}$ quadrant into $3^{\text {rd }}$ quadrant, $2^{\text {nd }}$ quadrant into $4^{\text {th }}$ quadrant, $3^{\text {rd }}$ quadrant into $3^{\text {rd }}$ quadrant, and $4^{\text {th }}$ quadrant into $4^{\text {th }}$ quadrant.

Line segment $-k<\operatorname{Re}(\xi) \leq 0$ and $0 \leq \operatorname{Re}(\xi)<k$ maps into $-k \leq \operatorname{Im}(\xi)<0$ (twice) i.e., $-k<\operatorname{Re}(\xi)<k$ maps into $-k \leq \operatorname{Im}(\xi)<0$ The positive and negative imaginary axes map into the ray $\operatorname{Im}(\xi)>-k$.

To evaluate

$w(x, y)=-\frac{1}{2 p} \mathrm{O}_{c} \frac{1}{\sqrt{x^{2}-k_{T}^{2}}} e^{-i x x-\sqrt{x^{2}-k_{T}^{2}} y} d x$,

we need a branch of multiple-valued function $f(x)=\sqrt{x^{2}-k_{T}^{2}}$, which has positive real part along the contour of integration. The only possible contour for the first branch $f_{1}(x)$ with the condition that $\operatorname{Re}\left(\sqrt{x^{2}-k_{T}^{2}}\right)>0$ is the contour as shown in figure 3 .

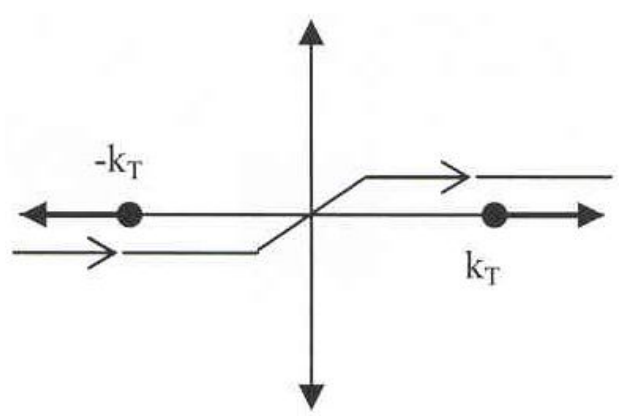

Fig. 3: Contour of $f_{1}(x)$ in the $\xi$-plane.

The only contour for the second branch $f_{2}(x)$ with the condition that $\operatorname{Re}\left(\sqrt{x^{2}-k_{T}^{2}}\right)>0$ is the contour as shown in Fig. 4. 


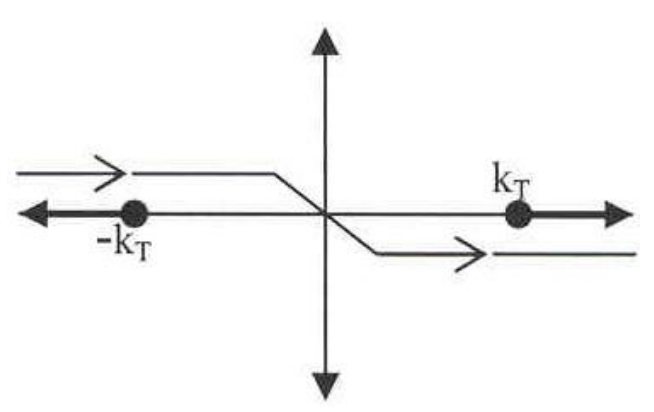

Fig. 4: Contour of $f_{2}(x)$ in the $x$-plane.

Hence, we conclude that $f_{2}(x)=\sqrt{r_{1} r_{2}} e^{\frac{i\left(q_{1}+q_{2}\right)}{2}}$ is an appropriate choice. Considering $x=r \cos \theta, y=r \sin \theta$ with $0<\theta<\pi$, the integral (Eq. 12) reduces as

$w(x, y)=-\frac{1}{2 p} \mathrm{ò}_{c} \frac{1}{\sqrt{x^{2}-k_{T}^{2}}} e^{-i r s} d x$

where $S=x \cos q-i \sqrt{x^{2}-k_{T}^{2}} \sin q$.

To apply Cagniard-de Hoop method, we need a contour $c_{1}$ in $\xi$-plane such that whenever $\xi$ is on $c_{1}, S$ must be real. Let us consider $0<q<p / 2, f_{2}(x)$ maps the first quadrant into the third quadrant and if $x=x_{1}+i x_{2}$, where $x_{1}>0, x_{2}>0$, then $\sqrt{x^{2}-k_{T}^{2}}=-x_{3}-i x_{4}$ for some real $x_{3}>0, x_{4}>0$, and

$S=x \cos q-i \sqrt{x^{2}-k_{T}^{2}} \sin q$

$S=\left(x_{1} \cos q-x_{4} \sin q\right)+i\left(x_{2} \cos q+x_{3} \sin q\right)$,

wherein the imaginary part on right hand side can not be equal to zero. Hence, we deduce that no part of $c_{1}$ can be in the $1^{\text {st }}$ quadrant. Similarly, it can also be shown that no part of $c_{1}$ can be in the $4^{\text {th }}$ quadrant. Hence, we conclude that if $c_{1}$ exists, it exists only in the left-half plane. Similarly, if $k_{1}>k_{T}>0$ and $x=x_{1}$ in the $2^{\text {nd }}$ quadrant then

$x_{1}=-k_{1} \cos q+i \sqrt{k_{1}^{2}-k_{T}^{2}} \sin q$,

which can be rewritten as

$\sqrt{x_{1}^{2}-k_{T}^{2}}=\sqrt{k_{1}^{2}-k_{T}^{2}} \cos q-i k_{1} \sin q$

thus,

$x_{1} \cos q-i \sqrt{x_{1}^{2}-k_{T}^{2}} \sin q=-k_{1}$.

Hence $c_{1}$ exist in the $2^{\text {nd }}$ quadrant and $S$ is negative along this contour. Similarly, taking $\xi=\xi_{2}$ in the $3^{\text {rd }}$ quadrant, it can be seen that $c_{1}$ exist in the $3^{\text {rd }}$ quadrant and $S$ is again negative along this contour.
Hence, we deduce that for $0<q<\frac{p}{2}, c_{1}$ is the branch

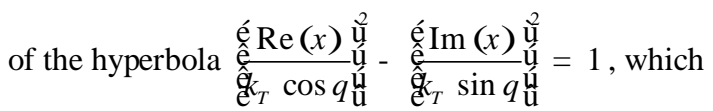
lies in the left-half plane as shown in figure 5.

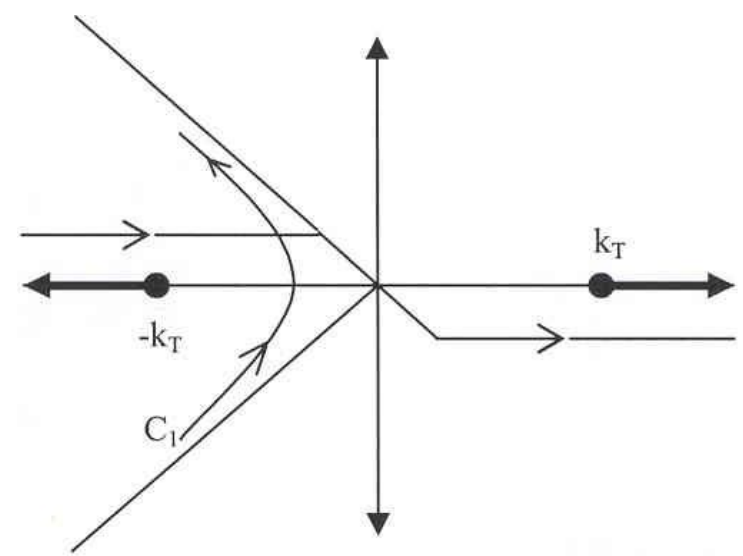

Fig. 5: Change of contour as $\mathrm{C}_{1}$ in the $\xi$-plane.

By considering appropriate arcs with large radii, contour $\mathrm{c}$ can be deformed as $c_{1}$. As radii $\rightarrow \infty$, the integration vanishes and consequently,

$$
\begin{aligned}
& w(x, y)=\frac{1}{2 p} \grave{\mathrm{O}}_{k_{T}}^{¥} \frac{1}{\sqrt{x_{1}^{2}-k_{T}^{2}}} \frac{\mathbb{I} x_{1}}{\operatorname{II} k_{1}} e^{i r k_{1}} d k_{1}+ \\
& \frac{1}{2 p} \underset{\ddagger}{\mathrm{O}_{T}} \frac{1}{\sqrt{x_{2}^{2}-k_{T}^{2}}} \frac{\mathbb{I} x_{2}}{\llbracket k_{1}} e^{i r k_{1}} d k_{1} .
\end{aligned}
$$

Considering $\sqrt{x_{1}^{2}-k_{T}^{2}}=\sqrt{k_{1}^{2}-k_{T}^{2}} \cos q-i k_{1} \sin q$ and $\sqrt{x_{2}^{2}-k_{T}^{2}}=-\sqrt{k_{1}^{2}-k_{T}^{2}} \cos q-i k_{1} \sin q$, the above integral reduces the solution as Hankel function of first kind $w(x, y)=\frac{i}{2} H_{0}^{(1)}\left(k_{T} r\right)$. Similarly, if $\frac{p}{2}<q<p$ then $c_{1}$ will be that branch of the hyperbola

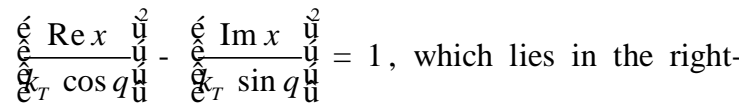
half plane and the end solution is Hankel function of first kind $w(x, y)=\frac{i}{2} H_{0}^{(1)}\left(k_{T} r\right)$.

\section{COMPARISON AND CONCLUSION}

Even earlier than 1973, Achenbach had investigated this problem of half-space subjected to anti-plane surface disturbance. He obtained a solution $w(x, y)=\frac{i}{2} H_{0}^{(2)}\left(k_{T} r\right)$. This solution was obtained by assuming the pivotal parameter $S=x \cos q-i \sqrt{x^{2}-k_{T}^{2}} \sin q$ to be real and positive. This parameter played significant role in applying the Cagniard-de Hoop method. 
In our analysis, investigations were carried out in detail about the choice of branch cuts, branches and their behavior in different quadrants of the $\xi$ - plane. Investigations show that the contour which simplifies the inversion integral, may or may not exist in some part of the $\xi$-plane and is very much dependent on the position of the point. To apply Cagniard-de Hoop method, the choice for the required contour $c_{1}$ to simplify the inversion integral depends particularly on $\theta$. In the half-plane, if $\frac{p}{2}<q<p, c_{1}$ must be selected as that branch of hyperbola which lies in left-half of $\xi$ plane. While for $\frac{p}{2}<q<p$, the contour $c_{1}$ must be selected as that branch of hyperbola which lies in righthalf of $\xi$-plane. Corresponding to the contour in figure 1 , the contour $c_{1}$ is as shown in figure 5 rather than as shown in figure 2 . The parameter $S$ is real but negative for all value of $0<q<p$. The ultimate solution is $w(x, y)=\frac{i}{2} H_{0}^{(1)}\left(k_{T} r\right)$.

In this paper, we have discussed an immensely important problem from applications prospective but unique in nature because it has an exact solution. Our investigations reflect that a good understanding of mathematical formulation and a considerable knowledge in applied mathematics are the essential ingredients to deal and understand the challenging phenomena of wave propagation.

\section{REFERENCES}

[1] J. D. Achenbach, "Wave Propagation in Elastic Solids", North-Holland. 1984.

[2] A. B. H. Love, "The Mathematical Theory of Elasticity", N.Y, Dover Publications, Inc, 1944.

[3] S. P. Timoshenko and J. N. Goodier, "Theory of Elasticity”, McGraw-Hill Co., 1987.

[4] K. B. Bullen, "An Introduction to the Theory of Seismology", Cambridge, University Press, 1963.

[5] W. M. Ewing, W. S. Jardetzky, and F. Press, "Elastic Waves in Layered Media", NY, McGraw-Hill Co., 1957.

[6] L. Cagniard, "Re'flexion et $\mathrm{Re}^{\prime}$ fraction des ondes Se'ismimique Progressives", Gauthiers-Villars, 1939.

[7] A. Schoch, "Schallreflexion, Schallbrechung und Schallbeugung", Ergebnisse der Exakten Naturwissenschaften, Vol. 23, Springer-Verlag, 1950.

[8] D. Gross, and Ch. Zhang, "On Wave Propagation in Elastic Solids with Cracks (Advance in Fracture Mechanics Series)", WIT press, 1997.

[9] J. Miklowitz, "Elastic Waves propagation, Applied Mechanics Surveys", Spartan Books, 1966

[10] B. A. Auld, "Acoustic Fields and Waves in Solids", Vol. I, Krieger, 1990.

[11] J. Billingham and A. C. King, "Wave Motion", Cambridge University Press, 2001.
[12] A. Bedford and D. S. Drumheller, "Introduction to Elastic Wave Propagation", John Wiley and Sons, 1994.

[13] H. Kolsky, "Stress Waves in Solids", NY, Dover Publications,Inc., 1963.

[14] L. Rayleigh, "The Theory of Sound”, Vol.(I \& II), N.Y, Dover Publications, Inc., 1945.

[15] L.M. Brekhovskikh, "Waves in Layer Media", NY, Academic Press, 1960.

[16] R. B. Lindsay, "Mechanical Radiation", NY. McGrawHill Co., 1960

[17] P.M. Morse and K.U. Ingard, "Theoretical Acoustics", NY, McGraw-Hill Co, 1968.

[18] I. Tolstoy and C. S. Clay, "Ocean Acoustics", NY, McGraw-Hill Co., 1966.

[19] J. L. Davis, "Mathematics of Wave Propagation", Princeton University Press, 2000.

[20] F. Moser, C. Valle, L. J. Jacobs, and J. Qu, “Application of Finite Element Methods to Study Transient Wave Propagation in Elastic Wave Guides", "Review of Progress in Quantitative NDE, Vol.16 A, 1998.

[21] F. Moser, L. J. Jacobs, and J. Qu, “Application of Finite Element Methods to Study Wave Propagation in Wave Guides", "NDT \& E Int., Vol. 32, 1999.

[22] B. Noble, "Wiener-Hopf Technique", Chelsea Pub.Co., 1988.

[23] A. T. de Hoop, "A Modification of Cagniard's method for Solving Seismic Pulse Problems", Applied Science Research, B 8, 349-356, 1960.

\section{BIOGRAPHY}

Dr. Mohammad Azram received his Bachelor degree in Mathematics from University of Peshawar, Pakistan in 1974. He obtained his Master degree in Mathematics with a position from University of Peshawar, Pakistan in 1976. He obtained his second Master degree in Mathematics from University of Idaho, USA in 1985 and then graduated with a Ph.D. from the same University in 1989. He started his career as a lecturer, Department of Mathematics, University of Peshawar, Pakistan in January 1977. He is now a professor in the Department of Mathematics, University of Peshawar, Pakistan. He has served the University of Peshawar in the capacity of Chairman, member Academic Council, member Board of Studies, member Affiliation Committee, member Peshawar University Teacher's Association, and Director of Postgraduate Programmes, Department of Mathematics, University of Peshawar, Pakistan.

Currently, he is a professor at Faculty of Engineering, IIUM, Malaysia. In addition, he is also Deputy Dean (Academic Affairs), Centre for Postgraduate Studies, IIUM, Malaysia. 